\title{
A importância da avaliação ergonômico-pedagógica de aplicativos educacionais e os desafios encontrados no campo da aprendizagem móvel
}

\author{
Raíza Portilho Nunes ${ }^{1}$, Isadora Mendes Santos ${ }^{1}$ \\ ${ }^{1}$ Faculdade de Sistemas de Informação - Universidade Federal do Pará (UFPA) \\ Cametá - PA - Brasil \\ $\{$ raisamendes 20, isadoramsantos $\}$ egmail.com
}

\begin{abstract}
This article discusses some problems that have been hindering the effectiveness of educational software evaluation, but which can still be minimized with future research in the area of computing. Through an ergonomic-pedagogical analysis of educational applications, the article aims to alert to the importance of the evaluation of educational software for the effectiveness of learning.
\end{abstract}

Resumo. Este artigo discute algumas lacunas que vêm impedindo a efetividade da avaliação de softwares educativos, mas que ainda podem ser minimizadas com pesquisas futuras na área de computação. Através de uma análise ergonômica-pedagógica de aplicativos educacionais para dispositivos móveis, busca-se alertar para a importância da avaliação de softwares educacionais para a efetividade do aprendizado.

\section{Introdução}

O uso de dispositivos móveis, principalmente smartphones, tem se incorporado cada vez mais ao cotidiano das pessoas, apresentando muitas oportunidades e desafios. O motivo para esse fato está na compreensão de que as tecnologias digitais móveis implicam em vantagens competitivas, conforto, mobilidade e autonomia para o usuário [Fonseca 2013]. Em conformidade com Gouvêa e Nakamoto (2015), apesar de existir uma grande diversidade de Softwares Educacionais (SE) disponíveis, nem todos propiciam a construção do conhecimento, ou nem apresentam fins pedagógicos, sendo meramente jogos de estímulos/respostas. Os autores ressaltam ainda que dos diversos modelos de avaliações existentes, a maioria preocupa-se em analisar somente o desempenho da ferramenta em si, desconsiderando a questão pedagógica do software. Salientando que os SE precisam ser produzidos por uma equipe multidisciplinar, com o auxílio de profissionais da educação, como pedagogos e professores e avaliados por uma comissão pedagógica.

Avaliar e classificar a qualidade e eficácia dos softwares educativos é uma tarefa delicada, que exige conhecimento prévio, apropriado e imparcial devido aos diversos pontos que devem ser analisados e as diversas características humanas de aprendizagem e motivação. Muitas abordagens para avaliação de SE já foram propostas em diversos estudos, mas esta pluralidade de métodos dificulta bastante a tarefa, principalmente pela ausência de padrões dos critérios e diferenças entre as abordagens. 
VII Congresso Brasileiro de Informática na Educação (CBIE 2018)

Anais do XXIX Simpósio Brasileiro de Informática na Educação (SBIE 2018)

Motivado por isso, este trabalho apresenta uma revisão das principais abordagens de avaliação de softwares educativos, e baseado nestas abordagens, propõe uma avaliação ergonômico-pedagógica de aplicativos relacionados às disciplinas abordadas no Exame Nacional do Ensino Médio (ENEM), na perspectiva de um profissional de pedagogia, objetivando aliar os conceitos desenvolvidos e consolidados nas pesquisas da área da educação com os da ergonomia de software.

Além desta seção, aborda-se na seção 2 a contextualização sobre a importância da avaliação de softwares educativos. A seção 3 apresenta algumas das principais abordagens de avaliação de softwares educativos. A seção 4 descreve a metodologia utilizada no trabalho. A seção 5 apresenta a avaliação, no contexto pedagógico e ergonômico, sobre as ferramentas escolhidas. E por fim, na seção 6 são apresentadas algumas considerações sobre o trabalho realizado e propostas para trabalhos futuros.

\section{A importância da avaliação de softwares educativos}

No contexto da educação, os softwares podem auxiliar os estudantes a assimilarem mais facilmente os conteúdos ministrados pelos professores em sala de aula, aplicar o conhecimento teórico através de aplicações práticas, permitir maior autonomia e controle do aluno com seu ritmo de aprendizagem e seus erros, e principalmente favorecer a adaptação aos diversos perfis de aprendizagem dos alunos [Brito Junior 2016]. Um software educativo possui características diferentes dos demais, já que sua ênfase é no aprendizado. Ele precisa estar inserido em um contexto pedagógico, que proporcione, entre outras coisas, a construção conhecimento, ou seja, além de ser eficiente, fácil de usar, ele deve ser também didático [Pinto e Cybis 2000].

De acordo com Silva e Fernandez (2007), ao desenvolver uma ferramenta para uso educativo é necessário atentar para três características essenciais: estimular o raciocínio e o pensamento crítico (minds-on), apresentar conteúdo relevante ao usuário (reality-on) e possibilitar a exploração (hands-on). Além dessas características básicas os autores destacam que somente inserir conteúdos originalmente impressos em papel em uma ferramenta digital não resultará em nenhuma vantagem significante na perspectiva didático-pedagógico. É preciso investigar e adaptar normas para guiar o desenvolvimento dessas ferramentas digitais, visando qualidade, eficiência e conformidade com as práticas educacionais, evitando ao máximo o surgimento de softwares inadequados e/ou pouco eficientes, que venham frustrar o usuário e a experiência da aprendizagem [Filho e Barbosa 2012].

Em conformidade com Pereira et al. (2016), a avaliação prévia deve identificar se o aplicativo em questão apresenta requisitos satisfatórios quanto aos aspectos ergonômicos e pedagógicos. Em termos conceituais, a ergonomia é um conjunto de conhecimentos científicos relativos ao homem e necessários para a geração de instrumentos e dispositivos que possam ser manuseados com o máximo de eficácia, segurança e conforto [Hack et al. 1999].

A ergonomia tem por objetivo avaliar as funcionalidades que estão relacionadas às necessidades dos usuários e avaliar os efeitos da interface sobre o usuário, que consiste na facilidade de aprendizagem do software e eficiência de uso. A pedagogia, por sua vez, é uma ciência aplicada constituída por outras ciências como a Filosofia, Epistemologia, Psicologia, Biologia, Economia e Ergonomia, dedicados à aprendizagem 
VII Congresso Brasileiro de Informática na Educação (CBIE 2018)

Anais do XXIX Simpósio Brasileiro de Informática na Educação (SBIE 2018)

que, como propriedade do processo pedagógico, é determinada por variados aspectos que resultam na interação sujeito/objeto sistema [Hack et al. 1999].

De acordo com Paiva (2002, apud Webber, Boff e Bono 2009), a avaliação de um software pode ser classificada como checklists, avaliação heurística, ensaios de interação, exploração cognitiva, questionários, entrevistas, inspeções formais de usabilidade, investigação contextual e pesquisas e a escolha de uma ou mais técnicas depende do tipo ou dos objetivos da avaliação pretendida pelo usuário. Entretanto, são poucas as diretrizes para aplicativos educativos para dispositivos móveis especificamente. Andrade, Junior e Silveira (2015), relatam que o estabelecimento de critérios e modelos para medir a qualidade de softwares educativos ainda não são muito comuns e que as métricas existentes utilizam metodologias não específicas, o que permite adaptações ao tipo de software avaliado, no caso, aplicativo para smartphone.

A partir disso, surge um grande desafio para a área da informática na educação: sobre qual o profissional mais adequado para realizar a avaliação, sobre o que deve ser avaliado no software, sob quais perspectivas se deve avaliar (qualidade de software, pedagógica, ou de usabilidade), e tudo isso levando em consideração a classificação do $\mathrm{SE}$, o público alvo, e a área de conhecimento.

\section{As diversas abordagens de avaliação de $\mathrm{SE}$ existentes e os desafios encontrados na avaliação de softwares móveis}

Conforme exposto anteriormente, há muitos anos estudam-se abordagens de avaliação de softwares educativos. Entretanto, a diversidade de abordagens propostas torna-se um desafio, principalmente nos tempos atuais, com o rápido avanço de tecnologias e suas diversas características.

Em 1989, Thomas Reeves propôs uma abordagem para avaliação de SE que lista, para análise, quatorze critérios pedagógicos e dez critérios sobre a interface. Cada critério é associado a uma escala bidirecional não numérica, que se utiliza de conceitos. Cabe ao avaliador verificar, para cada critério, como o SE se apresenta, e realizar uma marca sobre a escala. Quanto mais marcações à esquerda, maior é a fragilidade do SE em relação aos aspectos de usabilidade. Para os critérios pedagógicos, o diagnóstico é realizado pelo educador mediante análise da disposição dos pontos marcados na escala [Pereira et al. 2016].

A técnica de Mucchielli realiza a avaliação de um SE considerando o público alvo do mesmo e propõe um total de 10 critérios associados a uma escala numérica com pontuação variando entre 1 (um) e 5 (cinco). O resultado e o diagnóstico se dão pela análise da associação dos valores aos critérios, cuja apresentação é um conjunto de barras com os critérios e valores atribuídos a cada um. Essa técnica foi utilizada para avaliar distintos softwares educativos do tipo exercício, prática e simulação nas áreas de engenharia de software e multidisciplinar (matemática e português) mas a mesma não possui critérios que permitam a avaliação de aspectos pedagógicos e de uso do SE [Brito Junior 2016].

O instrumento de avaliação de LORI (Learning Object Review Instrument) de Nesbit, Belfer e Leacock (2003, apud Pereira et al. 2016), consiste em um checklist com um total de 9 critérios (1 pedagógico, 4 de qualidade de software e 4 de usabilidade). Os 
VII Congresso Brasileiro de Informática na Educação (CBIE 2018)

Anais do XXIX Simpósio Brasileiro de Informática na Educação (SBIE 2018)

critérios são associados a escalas numéricas de 5 pontos, com variação entre 1 (um) e 5 (cinco).

A FASE (Ferramenta Especialista para Avaliação de Software Educacional) [Webber, Boff e Bono 2009] constitui uma ferramenta web, com interface para usuários do tipo especialista e avaliador, banco de dados de questões, alternativas, atribuição de pesos a questões e alternativas e instrumento de avaliação com pontuação. Após o usuário preencher o instrumento de avaliação, a ferramenta retorna um feedback com as características do software, assim como a sua pontuação alcançada.

Vieira, Simões e Barreto (2012), avaliaram o software intitulado "É o Bicho", que é direcionado à disciplina da matemática, proposta para o nível de segundo ano do ensino fundamental I. Os quesitos observados para análise e discussão, tomaram por base critérios descritos em uma tabela adaptada pelo pesquisador, onde o critério avaliativo foi a observação e mensuração do domínio de cada quesito ergonômico e pedagógico, como facilidade dos procedimentos e eficiência de uso, por exemplo. Mediante o procedimento do manuseio prático do funcionamento e da dinâmica do software, o avaliador verifica a existência do quesito e o grau de satisfação é dado por um conceito que pode ser desde $\mathrm{P}$ (péssimo) até $\mathrm{E}$ (excelente).

A Ferramenta Pedagógica para Avaliação Ergonômica de Software Educativo (Pedagogical Ergonomic Tool for Educational Software Evaluation - PETESE) [Coomans e Lacerda 2015], consiste em um guia de usabilidade pedagógica para software matemático. Ela ordena os critérios em 6 aspectos diferentes: aspectos técnicos da construção do sistema, caráter pedagógico, interface do produto, conteúdo do software e aspectos de usabilidade.

Barbosa, Oliveira e D’Carlo (2016), apresentaram um conjunto de heurísticas específico para avaliar e caracterizar a usabilidade de aplicativos móveis educacionais, que visa cobrir as lacunas que as heurísticas existentes na literatura não conseguem avaliar em relação à usabilidade desse domínio de aplicação. Além disso, o conjunto de heurísticas proposto poderá ser utilizado tanto para aplicativos educacionais já desenvolvidos, quanto para aplicativos em fase de desenvolvimento.

Os trabalhos pesquisados demonstram a falta de padronização dos métodos de avaliação, deixando clara uma grande diferença entre os tipos de avaliações, suas formas de aplicação, além da quantidade e descrição dos critérios utilizados e a forma de apresentação e análise de resultados.

Nenhum trabalho apresenta uma abordagem que englobe aspectos de qualidade de software, pedagógicos e ergonômicos. Enquanto alguns avaliam apenas aspectos de usabilidade, outros levam em conta apenas normas técnicas de avaliação do processo e produto, e não observam características pedagógicas, essenciais para os fins que foram desenvolvidos.

Poucas diretrizes foram encontradas na literatura para embasar avaliações de aplicações móveis, já que estas possuem outras características como mobilidade, e ubiquidade, além de que por serem mais atuais, se direcionam a um público alvo mais dinâmico e exigente. Grande parte dos métodos de avaliação são antigas e voltadas para softwares para computadores desktop, o que faz com que os critérios em alguns casos 
VII Congresso Brasileiro de Informática na Educação (CBIE 2018)

Anais do XXIX Simpósio Brasileiro de Informática na Educação (SBIE 2018)

não se apliquem à aprendizagem móvel, bem como não consideram critérios extremamente importantes inerentes aos aplicativos móveis.

\section{Metodologia}

Quanto à sua natureza, esta pesquisa caracteriza-se como aplicada, por ser dirigida à compreensão de um problema real, onde o conhecimento gerado deverá servir de estímulo à prática de análise de aplicativos educativos. Caracteriza-se também como exploratória, ao proporcionar uma visão geral sobre abordagens para avaliação de softwares educativos, baseando-se em levantamento bibliográfico.

Considerando que o enfoque desta investigação está centrado na análise de duas propriedades, uma de caráter ergonômico (usabilidade) e outra de caráter pedagógico (aprendizagem), e ressaltando a carência de um padrão único de princípios ergonômicos estabelecidos para os aplicativos móveis educacionais, foram listados neste trabalho os requisitos ergonômicos e pedagógicos que aparecem com mais frequência entre os demais pesquisadores na área. Tais requisitos serão verificados nos aplicativos analisados neste documento. Além disso, o foco desta pesquisa não é criticar ou destacar aspectos negativos das ferramentas selecionadas, mas sim despertar e estimular a necessidade de se desenvolver produtos dessa natureza guiados por aspectos essenciais para a efetivação da qualidade no aprendizado proposto. Os aspectos ergonômicos considerados neste trabalho são:

Controle do Usuário: Avalia as possibilidades de o usuário controlar o encadeamento e a realização das ações;

Mensagens de Erro: As mensagens de erro do aplicativo devem possuir uma redação simples e clara ao invés de intimidar o usuário;

Saídas Claramente Demarcadas: O usuário controla o sistema. Ele pode, a qualquer momento, abortar uma tarefa, ou desfazer uma operação e retornar ao estado anterior;

Flexibilidade e eficiência de uso: O software precisa ser flexível, ou seja, ser familiar para usuários avançados e ao mesmo tempo ser fácil para usuários leigos;

Design estético e minimalista: Deve-se apresentar exatamente a informação que o usuário precisa no momento, nem mais nem menos;

Diálogos simples e naturais: Os "diálogos" do software precisam ser simples, diretos, naturais, e presentes nos momentos em que são necessários;

Ajuda e documentação: O ideal é que um software seja tão fácil de usar (intuitivo) que não necessite de ajuda ou documentação. Mas se for necessária, a ajuda deve estar disponível e facilmente acessível;

No que se refere à avaliação pedagógica foi adotada uma tabela-modelo para classificar a qualidade de aplicativos adaptada de [Vieira, Simões e Barreto 2012], que avalia as abordagens pedagógicas que um software educacional deve considerar. Os principais requisitos pedagógicos pontuados pelos autores são:

Harmonia entre as atividades: Quando o material pedagógico inserido nas atividades está condizente com os objetivos e adequado à faixa etária proposta;

Clareza e Interatividade: Quando o usuário é orientado passo a passo nos procedimentos a serem seguidos; 
VII Congresso Brasileiro de Informática na Educação (CBIE 2018)

Anais do XXIX Simpósio Brasileiro de Informática na Educação (SBIE 2018)

Facilidade dos Procedimentos: A maneira como os processos estão apresentados, minimizando confusões, dúvidas, insatisfação ou frustração no usuário;

Aspectos Motivadores: Recursos presentes na ferramenta para estimular o aluno durante o aprendizado;

Oportunidade de retornar à atividade em caso de erro: Oferecer ao usuário a possibilidade de retornar à questão respondida de forma errada anteriormente;

Atividades Reflexivas: Oportunidade de reflexão e interpretação que a atividade oferece ao usuário;

Feedback: Oferecer retorno da avaliação do desempenho do usuário sobre ações ou resultados obtidos.

Atualmente encontra-se uma diversidade de aplicativos de conteúdo educativo para os mais variados contextos e necessidades da educação, agradáveis e adaptáveis a muitos perfis de usuários, desde jogos, simulados online até realidade aumentada. Todavia, este trabalho aborda apenas aplicativos de m-learning específicos para a preparação para o ENEM.

Os aplicativos foram selecionados em duas etapas: geral e específica. Para a primeira etapa foram apurados os aplicativos resultantes da pesquisa por "ENEM" no buscador da Play Store, totalizando 180 aplicativos nos resultados da busca. Em seguida, os aplicativos da etapa geral foram analisados a fim de verificar suas funcionalidades, para que se pudesse selecionar os aplicativos com maior número de funcionalidades, como exercícios, simulados, resumos, jogos e conteúdo, pois acreditase que, além de chamarem mais atenção dos estudantes pela diversidade de metodologias, tornaria a avaliação mais consistente.

Após esta separação, levou-se em consideração os aplicativos que possuíam o maior número de downloads e melhor avaliação pelos usuários (entre quatro e cinco estrelas) até a data do trabalho. Nesta etapa foram selecionados os 3 (três) aplicativos para análise.

Não se considerou analisar apenas aplicativos gratuitos ou comerciais, entretanto, os aplicativos pagos normalmente são menos baixados, o que fazia com que estes nem passassem no critério de maior número de downloads, e acredita-se ser importante considerar o número de downloads pois representa o que o público está realmente buscando e utilizando, e analisar a qualidade de softwares já disseminados entre o público é importante, principalmente, para chamar atenção para a real efetividade do aprendizado através destes aplicativos e estimular a avaliação destas ferramentas, até mesmo pelos próprios desenvolvedores.

Uma observação relevante a ser mencionada é o fato de que os aplicativos disponíveis para download analisados nas etapas da pesquisa, aparentemente não foram desenvolvidos sob a orientação de especialistas da educação, e se houve consulta aos profissionais de ensino, este fato não foi referenciado nas páginas de download dos aplicativos.

\section{Resultados e Discussão}

Com base nos requisitos pedagógicos citados na seção anterior, obteve-se o resultado da avaliação. O primeiro aplicativo a ser analisado foi o Study Enem, seguido do Simulado Enem 2017 e Enem 2017 Simulado Gabaritando. 
VII Congresso Brasileiro de Informática na Educação (CBIE 2018)

Anais do XXIX Simpósio Brasileiro de Informática na Educação (SBIE 2018)

Inicialmente o pedagogo fez uma exploração individual em cada aplicativo para identificar aspectos básicos como o tipo de abordagem e proposta educativa. Em seguida foram anotados os quesitos observados para avaliação, tomando por base critérios adaptados de [Vieira, Simões e Barreto 2012] mostrados na Tabela 1. Após a análise prévia foram atribuídos conceitos referentes ao grau de satisfação aos requisitos avaliados, em que E significa excelente; B significa bom; R representa regular; e P quer dizer que é péssimo.

Tabela 1. Resultado da avaliação do profiss ional pedagogo

\begin{tabular}{|c|c|c|c|}
\hline & $\begin{array}{l}\text { Study } \\
\text { Enem }\end{array}$ & $\begin{array}{c}\text { Simulado } \\
\text { Enem } 2017\end{array}$ & $\begin{array}{c}\text { Enem } 2017 \\
\text { Simulado } \\
\text { Gabaritando }\end{array}$ \\
\hline \multicolumn{4}{|c|}{ Aspectos Ergonômicos } \\
\hline Controle do usuário & $\mathrm{B}$ & $\mathrm{R}$ & $\mathrm{R}$ \\
\hline Mensagens de erro & $\mathrm{R}$ & $\mathrm{R}$ & B \\
\hline Saídas claramente demarcadas & $\mathrm{R}$ & $\mathrm{B}$ & $\mathrm{R}$ \\
\hline Flexibilidade e eficiência de uso & $\mathrm{E}$ & $\mathrm{E}$ & $\mathrm{E}$ \\
\hline Design estético e minimalista & $\mathrm{R}$ & B & B \\
\hline Diálogos simples e naturais & $\mathrm{E}$ & $\mathrm{E}$ & $\mathrm{E}$ \\
\hline Ajuda e documentação & $\mathrm{R}$ & $\mathrm{R}$ & $\mathrm{R}$ \\
\hline \multicolumn{4}{|c|}{ Aspectos Pedagógicos } \\
\hline Harmonia entre as atividades e os objetivos & $\mathrm{E}$ & $\mathrm{B}$ & $\mathrm{B}$ \\
\hline Clareza e interatividade & $\mathrm{R}$ & $\mathrm{B}$ & $\mathrm{B}$ \\
\hline Facilidade dos Procedimentos & $\mathrm{R}$ & $\mathrm{B}$ & $\mathrm{R}$ \\
\hline Aspectos motivadores & $\mathrm{B}$ & $\mathrm{B}$ & $\mathrm{E}$ \\
\hline Oportunidade de retornar à atividade em caso de erro & $\mathrm{P}$ & $\mathrm{P}$ & $\mathrm{P}$ \\
\hline Atividades reflexivas & $\mathrm{R}$ & $\mathrm{R}$ & $\mathrm{B}$ \\
\hline Feedback & $\mathrm{B}$ & $\mathrm{R}$ & $\mathrm{B}$ \\
\hline
\end{tabular}

A avaliação do profissional de pedagogia concluiu que os aplicativos analisados trabalham com pouca estrutura e exploram superficialmente os conceitos referentes aos temas abordados, além de comumente adotarem metodologias baseadas em memorização e fragmentação do conhecimento. Feedback do desempenho, harmonia 
VII Congresso Brasileiro de Informática na Educação (CBIE 2018)

Anais do XXIX Simpósio Brasileiro de Informática na Educação (SBIE 2018)

entre as atividades e retorno ao aluno sobre a realização de sua atividade são características fundamentais encontradas nos aplicativos. Atividade de análise e investigação e oportunidade de retornar à atividade em caso de erro são quesitos que necessitam de mais atenção por parte dos desenvolvedores.

Quanto ao resultado dos aspectos ergonômicos avaliados nos aplicativos, podese perceber que as ferramentas digitais atendem satisfatoriamente aos quesitos abordados, possuem capacidade de serem manuseadas por diferentes perfis de usuários, além de apresentarem saídas claramente demarcadas e proporcionarem controle dos procedimentos aos usuários, o que configura adequação à tarefa, pois o design estético e minimalista bem como diálogos simples e naturais implicam na satisfação do usuário.

A quantidade de recursos que os aplicativos solicitam de seus usuários (como esforço cognitivo e tempo) para a satisfação de seus objetivos com as ferramentas é um quesito a ser revisto. Outro aspecto de extrema importância em uma ferramenta digital é a ajuda e documentação, atentando primeiramente que a ergonomia evita ao máximo a necessidade de ajuda na utilização do software. Ainda assim, disponibilizar documentação e ajuda deve ser necessário para orientar o usuário em caso de dúvida, também deve ser visível e de fácil acesso.

\section{Considerações Finais}

Este trabalho teve como objetivo apresentar uma revisão das principais abordagens de avaliação de softwares educativos, e baseado nestas abordagens, realizar uma avaliação ergonômico-pedagógica de aplicativos relacionados às disciplinas abordadas no Exame Nacional do Ensino Médio (ENEM), na perspectiva de um profissional de pedagogia, intentando verificar a qualidade dos conteúdos dispostos em aplicativos educativos. A expectativa é estimular o olhar crítico com relação a disposição de conteúdo educativo em ferramentas digitais, pois, tendo em vista a grande diversidade de materiais educacionais informatizados cabe aos desenvolvedores e profissionais da educação questionar se esses programas realmente agregam valor ao aprendizado do usuário.

Desta forma, levantou-se alguns argumentos para expressar a importância da avaliação de softwares educativos, em virtude da necessidade de cuidados constantes dos profissionais da tecnologia e da educação e as competências necessárias para propagar informação de qualidade através da tecnologia digital. Esses cuidados, referem-se principalmente a requisitos ergonômicos e pedagógicos. Ergonômicos para que o usuário, consiga utilizar os recursos do software com o máximo de segurança, conforto e produtividade e, pedagógicos, para que as estratégias de ensino estejam em conformidade com o objetivo educacional e as necessidades do aprendiz.

Entretanto, como observado nas seções anteriores, apesar de haver diversas abordagens e métricas para avaliar a qualidade de softwares, a falta de padronização configurou-se como um desafio para a realização deste trabalho. Isso porque a profusão de normas e guias dificulta a tarefa de avaliar pela vastidão de parâmetros, escalas diferentes, diagnósticos com distintos formatos, diversidade dos critérios definidos nas diferentes abordagens, e confusão na especificação do tipo de software que as normas se aplicam. De acordo com Pereira et al. (2016), por mais que seja utilizada uma abordagem mista na avaliação, adaptando critérios de diferentes métricas, as premissas cognitivas e de expertise necessárias por parte do avaliador podem dificultar o processo 
VII Congresso Brasileiro de Informática na Educação (CBIE 2018)

Anais do XXIX Simpósio Brasileiro de Informática na Educação (SBIE 2018)

avaliativo. Nenhum trabalho apresenta uma abordagem que englobe aspectos de qualidade pedagógicos e ergonômicos em aplicativos para dispositivos móveis, por exemplo. Por esse motivo foi necessário reunir e adaptar os requisitos ergonômicos e pedagógicos mais recorrentes nos trabalhos pesquisados cabíveis ao tipo de software desejado.

Espera-se com esse trabalho estimular pesquisas futuras para a criação de critérios específicos e unificados para cada tipo de software, ou para cada disciplina ou público alvo para gerar uma padronização na avaliação e facilitar a comparação entre os softwares. Reitera-se que um dos objetivos motivadores deste trabalho é propor e incentivar o uso de métricas, normas e guias de avaliação da qualidade do conteúdo e abordagem ergonômica e pedagógica adotada nessas ferramentas digitais, pois desconsiderar tais aspectos implica em softwares pouco eficazes no processo educativo de seus usuários.

Para as próximas etapas desta pesquisa pretende-se fazer uma avaliação dos aplicativos educativos na concepção dos usuários finais, ou seja, os aprendizes que efetivamente utilizam tais softwares. Além disso, pretende-se realizar a avaliação com outros profissionais da educação com a finalidade de comparar as análises e discutir as discrepâncias para obter resultados mais significativos.

\section{Referências}

Andrade, M. V. M., Araújo Jr., C. F. e Silveira, I. F. (2015). "Critérios de qualidade para aplicativos educacionais no contexto dos dispositivos móveis (M-Learning)". Em Nuevas Ideas em Informática Educativa. Congresso Internacional sobre Informática na Educação (TISE).

Barbosa, G.; Oliveira, E.; D’Carlo, D. (2016) “Usabilidade em aplicativos móveis educacionais: Um conjunto de heurísticas para avaliação". Anais do XXVII Simpósio Brasileiro de Informática na Educação, p. 777-786.

Brito Junior, O. D. O. (2016) “Abordagens Para Avaliação De Software Educativo E Sua Coerência Com Os Modelos De Qualidade De Software Abordagens Para Avaliação De Software Educativo E Sua Coerência Com Os Modelos De Qualidade De Software.” [S.L.] Universidade Federal Da Paraíba, 2016.

Coomans, S.; Lacerda, G.S. (2015). "PETESE, a Pedagogical Ergonomic Tool for Educational Software Evaluation". Procedia Manufacturing 3, 5881-5888.

Filho, N. F. D. e Barbosa, E. F. (2012). "Estudo e Definição de um Conjunto de Características e Requisitos para Ambientes de Aprendizagem Móvel São Carlos". Anais do $23^{\circ}$ Simpósio Brasileiro de Informática na Educação (SBIE 2012), ISSN 2316-6533. Rio de Janeiro-Rj, Brasil.

Fonseca, A. G. M. da. (2013). "Aprendizagem, mobilidade e convergência: mobile learning com celulares e smartphones”. Em: Revista_Mídia_e_Cotidiano 2 (2), 265283, 2013.

Gouvêa, M. C M e Nakamoto, P. T. (2015). "Avaliação de Software Educacional: Uma Oportunidade de Reflexão da Educação na Sociedade do Conhecimento.”. Em III Congresso Internacional Trabalho Docente e Processos Educativos. UNIUBE, 2015. 
VII Congresso Brasileiro de Informática na Educação (CBIE 2018)

Anais do XXIX Simpósio Brasileiro de Informática na Educação (SBIE 2018)

Hack, C. A., Souza, C. F. P., Thomé, A. C., Corrêa, Z. R. e Abreu, C. W. (1999). "Ergonomia Em Software Educacional: A possível integração entre Usabilidade e aprendizagem". Em Atas Ihc 99 - UNICAMP. São Paulo-SP, Brasil.

Nesbit, J. C., Belfer, K., \& Leacock, T. (2003). "Learning object review instrument (LORI)". E-learning research and assessment network.

Paiva, C.R. (2002). "Avaliação De Software Educativo História Do Mundo, Uma Aventura Visual: Aplicações No Ensino Presencial De História”. Dissertação de Mestrado. Florianópolis: UFSC, 2002.

Pereira, W. S., Silva, R. S. T. Da., Filho, R. J. C., Silva, W. R. De A., Aguiar, Y. P. C., Dantas, V. F. (2016). "Avaliação de Software Educativo: Análise de Abordagens para Definição de Diretrizes”. Em Nuevas Ideas en Informática Educativa, Volume 12 , p. $557-562$.

Pinto Andres, D.; Cybis, W. De A. (2000) "Um Estudo Teórico Sobre As Técnicas De Avaliação De Software Educacional." Em VI Congreso Argentino De Ciencias De La Computación.

Silva, R. M. D. Da. E Fernandez, M. A. (2007). "Recursos informáticos projetados para o ensino de ciências: bases epistemológicas implicadas na construção e desenvolvimento de objetos de aprendizagem." Em Objetos de Aprendizagem: uma proposta de recurso pedagógico. Organização: Prata, C. 1; Nascimento, A. C. A. A. Brasília: MEC, SEED.

Vieira, M. M. S.; Simões, L. L. F. e Barreto, A. L. de O. (2012). "Avaliação De Software Educativo: Aspectos Pedagógicos E Técnicos." Em Revista Faculdade Cearense (FAC). Vol. 5 - $\mathrm{n}^{\circ} 1 / 2012$.

Webber, C.; Boff, E.; Bono, F. (2009) "Ferramenta Especialista Para Avaliação De Software Educacional". XX Simpósio Brasileiro De Informática Na Educação. 